\title{
Modeling Adsorption and Transport of Chrome VI onto Iron Oxide-Coated Sand filter media
}

\section{Fulbert TOGUE KAMGA ${ }^{1,2 *}$, Hélé Raïssa MUKAM MAGNE${ }^{1}$, Calvia MADIE YONTI ${ }^{1}$, André CHAMGOUE CHEAGE ${ }^{3}$, César MBANE BIOUELE}

\author{
${ }^{1}$ Environmental physics laboratory, Faculty of Science, University of Yaoundé I, Box 812 Yaoundé, Cameroon \\ ${ }^{2}$ Institute of Fisheries and Aquatic Sciences at Yabassi, University of Douala, Box 2701 Douala, Cameroun \\ ${ }^{3}$ Department of Physics, University of Yaounde I, Box 812 Yaounde, Cameroon \\ * Correspondence: kamgafulbert@yahoo.fr; Tel.: +237 690980854
}

\begin{abstract}
Natural decay of pollutants is rarely considered in mass transport equation. This paper develops a mathematical model of a filter based on iron oxide-coated sand for the removal of chromium in groundwater and surface water. The natural decay of chromium was analyzed using a zero-order decay reaction. The advection dispersion equation for this purpose is solved analytically using the Laplace transform method and numerically by the Fourth order Runge kutta method to determine the spatio-temporal distribution of the pollutant through the filter. The control parameters are the adsorption coefficient, the initial concentration and the chromium degradation coefficient. It has been proven that the chromium degradation coefficient strongly affects the concentration values of pollutants inside the filter. The results of this work should be used to manufacture low-cost filters based on iron oxide-coated sand.
\end{abstract}

Keywords- Filter, Sand, Iron oxide, Chromium, Adsorption, Advection-dispersion, Laplace.

\section{INTRODUCTION}

Water is an essential component in the universe and plays an important role in the proper functioning of ecosystems [1]. Despite this, access to drinking water is an increasingly recurrent challenge worldwide and it is estimated that nearly eight hundred and eighty (880) million people lack safe drinking water at home $[2,3]$. Water pollution results mainly from poorly planned urban and rural development, industrial discharges, agricultural discharges, geological and environmental activities, domestic activities etc., it is a serious problem because it affects our lives [4]. In addition, the World Health Organization (WHO) estimates that $80 \%$ of the diseases that affect the population are directly carried by water: 400 million people are permanently affected by gastroenteritis, 160 million from malaria and 30 million onchocerciases [5]. The industry uses heavy metals in various fields because they have a high electrical www.ijeab.com conductivity; however, these heavy metals have some toxicity to humans, leading in particular toxic effects on the nervous system, carcinogenic effects on the blood and the bone marrow and kidney disorders.

Chromium is one of the most widely used metals in industries, since it has enough advantages for tanneries, textile, wood processing, agribusiness... Chromium VI is the most problematic form of chromium since in this form chromium is very toxic and very soluble in water. This solubility gives it great mobility in ecosystems. Today, following a lack of compliance with laws or accidents, a large number of industrial sites are polluted by chromium. Therefore, the development of low-cost, easy-to-maintain technologies to reduce this pollutant in drinking water sources to an acceptable level for consumption is an interesting research topic.

The methods implemented today to treat discharges polluted by chromium (VI) are generally aimed at recovering this metal, in order to reuse it and thus reduce its ecotoxicological impact. In parallel, physicochemical methods attempt to eliminate $\mathrm{Cr}(\mathrm{VI})$ and electrochemical methods attempt to reduce $\mathrm{Cr}$ (VI) to $\mathrm{Cr}$ (III). Iron oxide-coated sand has proven its effectiveness for the elimination of various heavy metals $(\mathrm{As}, \mathrm{Cd}, \mathrm{Cr}, \mathrm{Pb}, \ldots)$ at an elimination rates close to $100 \%$. In this study, we develop a mathematical model to understand and predict the adsorption of chromium VI in a fixed column bed made witch iron oxide-coated sand. As Chromium undergoing a natural decay, the discussion will be focus on the effect of the degradation coefficient $\lambda$ on the spatio-temporal variation of the pollutants inside the filter.

\section{MATERIAL AND METHODS}

\subsection{Preparation of iron oxide-coated sand}

The iron oxide-coated sand was prepared using a procedure similar to that of Bailey et al. (1992) [6]. The author used washed and dried river sand with a weight of $200 \mathrm{~g}$ and a geometric size of $0.49 \mathrm{~mm}$ [7]. It is obtained by mixing for 2 
$\min , 80 \mathrm{ml}$ of a solution of ferric nitrate $2 \mathrm{M}\left(\mathrm{Fe}\left(\mathrm{NO}_{3}\right)\right.$ $\left.39 \mathrm{H}_{2} \mathrm{O}\right)$ to washed river sand and dried. He then placed the mixture in a drying oven at $110^{\circ} \mathrm{C}$ for 14 hours. He finally places the mixture in distilled water until the runoff is cleaned at $105^{\circ} \mathrm{C}$ and stored in closed bottles [7].

\subsection{Mathematical description}

The linear dispersion advection-equation is used to describe the transport of pollutants in this fixed column bed (Fig. 1). The equation was derived to predict aqueous concentrations of contaminants over time and space in the porous medium. It is written as follows: [8]

$\rho \frac{\partial q}{\partial t}+\theta \frac{\partial c}{\partial t}=\theta \mathrm{D} \frac{\partial^{2} \mathrm{c}}{\partial \mathrm{x}^{2}}-\mathrm{v} \frac{\partial \mathrm{c}}{\partial \mathrm{x}}$

Where $\rho$ is the density, $\theta$ the water content, $D$ the hydrodynamic dispersion coefficient, V: average Darcy velocity and $q$ the equilibrium adsorbed concentration which is described as follows [8]:

$q=k c^{n}$

Where $\mathrm{k}$ is the adsorption capacity and $\mathrm{n}$ the adsorption intensity.

In the absence of the iron oxide-coated sand which is the adsorbent, $\mathrm{Cr}$ (VI) ion disappeared naturally from the solution. Given the fact that chromium is a naturally decreasing pollutant, this dis appearance rate was assumed to be at zero order as follows:

$-d c / d t=\lambda ; c=c_{0}$ at $t=0$

Where $\lambda$ is the zero order rate constant or chromium degradation coefficient, $c_{0}$ is the initial $\mathrm{Cr}(\mathrm{VI})$ concentration and $\mathrm{c}$ the $\mathrm{Cr}(\mathrm{VI})$ concentration at the time $\mathrm{t}$.

By adding a decay term $\lambda c$ to the equation above according to Runkel, 1996 and by considering a linear adsorption is otherm $(\mathrm{n}=1)$, after a few transformations, we obtain:

$\frac{\partial c}{\partial t}=d \frac{\partial^{2} \mathrm{c}}{\partial \mathrm{x}^{2}}-\mathrm{u} \frac{\partial \mathrm{c}}{\partial \mathrm{x}}-\lambda c$

The appropriate boundary conditions for the model are given by:

$$
\begin{aligned}
c(\mathrm{x}, 0)=0, \mathrm{x} \geq 0 ; \\
c(\infty, 0)=0, \mathrm{x} \geq 0
\end{aligned}
$$

\subsection{Analytical solution of the mathematical model}

The analytical solution is obtained using Laplace transform. It is use to transform one or more partial derivatives of the differential equation into algebraic expressions. We call Laplace transform of the function $G$ denoted $L$ (G), the function of complex variable $\mathrm{P}$ defined by:

$\mathrm{L}[\mathrm{G}(\mathrm{t})]=\bar{G}(\mathrm{p})=\int_{0}^{\infty} e^{-P t} G(x, t) d t$

Where $\mathrm{L}$ denotes the Laplace transform, $\mathrm{G}(\mathrm{t})$ is a function of time, $\bar{G}(P)$ is a function of the transformed space, it corresponds to the Laplace value. The operational property of the transformation that eliminates a partial derivative of the equation is as follows:

$L \frac{\partial G(t)}{\partial t}=p \bar{G}-G(0)$

Where $G(0)$ is the value of $G$ at time $t=0$. To reduce equation (4) to a more familiar form, we take:

${ }_{c}(x, t)=\Gamma(x, t) \exp \left[\frac{u x}{2 d}-\frac{u^{2} t}{4 d}-\lambda^{\prime} t\right]$

Let $\emptyset(x, t)=\frac{u x}{2 d}-\frac{u^{2} t}{4 d}-\lambda^{\prime} t$

By substituting equation (8) in equation (4), we obtain:

$\frac{\partial \Gamma}{\partial t}=d \frac{\partial^{2} \Gamma}{\partial x^{2}}$

The initial and boundary conditions (5) become:

$c(\mathrm{x}, 0)=0, \mathrm{x} \geq 0$

$c(0, t)=c_{0} \exp \left(\frac{u^{2} t}{4 d}+\lambda^{\prime} t\right), \mathrm{t} \geq 0$

$c(\infty, 0)=0, \mathrm{x} \geq 0$

The Laplace transform of equation (9) is:

$L\left(\frac{\partial \Gamma}{\partial t}\right)=d \frac{\partial^{2} \Gamma}{\partial x^{2}}$

Therefore, it's reduced to an ordinary differential equation

$\frac{\partial^{2} \bar{\Gamma}}{\partial x^{2}}=\frac{P}{d} \bar{\Gamma}$

The solution of the problem is:

$\Gamma(x, t)=\frac{c_{0}}{2} \exp \left[\frac{u^{2} t}{4 d}+\lambda^{\prime} t\right]\left[e^{2 \varepsilon} \operatorname{erfc}\left(\alpha+\frac{\varepsilon}{\alpha}\right)+\right.$

$\left.e^{-2 \varepsilon} \operatorname{erfc}\left(\alpha-\frac{\varepsilon}{\alpha}\right)\right]$

Substituting (13) in equation (8), the solution becomes:

$\frac{c}{c_{0}}=\frac{1}{2} \exp \left[\left(\frac{u x}{2 d}\right) e^{-2 \varepsilon} \operatorname{erfc}\left(\alpha-\frac{\varepsilon}{\alpha}\right)+e^{2 \varepsilon} \operatorname{erfc}\left(\alpha+\frac{\varepsilon}{\alpha}\right)\right]$

Where, $\alpha=\frac{x}{2 \sqrt{d t}}, \varepsilon=\sqrt{\left(\frac{u^{2}}{4 d}+\lambda^{\prime}\right)}\left[\frac{x}{2 \sqrt{d}}\right], H=\frac{2 \lambda^{\prime} d}{u^{2}}$ and $\Gamma=$ $\sqrt{1+2 H}$

Finaly:

$$
\begin{aligned}
& \quad \frac{c}{c_{0}}=\frac{1}{2}\left\{\exp \left[\frac{u x}{2 d}(1-\Gamma)\right] \operatorname{erfc}\left(\frac{x-u t \Gamma}{2 \sqrt{d t}}\right)+\exp \left[\frac{u x}{2 d}(1+\right.\right. \\
& \left.\left.\Gamma) \operatorname{erfc}\left(\frac{x+u t \Gamma}{2 \sqrt{d t}}\right)\right]\right\}
\end{aligned}
$$

\subsection{Numerical solution of the mathematical model}

The numerical solution allows to take into account more complex heterogeneous systems, and they can be thus more realistic and flexible to simulate the real conditions. It is not very obvious to obtain an analytical solution of linear PDE such as equation (4). However, there is an approximate solution for linear PDEs obtained from different numerical methods. Therefore, to solve the EDP of pollutant transport in porous media, we use numerical methods. The partial discretization method, where the finite difference approximation is used to obtain the discrete form of the spatial derivative. Then, the finite space difference diagram in space is used to have discrete shape spatial derivation equations of equation (4). Finally, the fourth order Runge Kutta system (RK4) is used to solve equation (4). This 
numerical technique is used to solve the ordinary differential equation expressed as follows:

$\frac{\partial c}{\partial t}=g(t, c)$

The RK4 algorithm is written as follows:

$\mathrm{k}_{1}=\Delta \mathrm{t} \mathrm{g}(\mathrm{t}, \mathrm{c}(\mathrm{t}))$

$\mathrm{k}_{2}=\Delta \mathrm{t} \quad \mathrm{g}\left(\mathrm{t}+\frac{1}{2} \Delta \mathrm{t}, \mathrm{c}(\mathrm{t})+\frac{1}{2} \mathrm{k}_{1}\right)$

$\mathrm{k}_{3}=\mathrm{g}\left(\mathrm{t}+\frac{1}{2} \Delta \mathrm{t}, \mathrm{c}(\mathrm{t})+\frac{1}{2} \mathrm{k}_{2}\right)$

$\mathrm{k}_{4}=\Delta \mathrm{t} \quad \mathrm{g}\left(\mathrm{t}+\Delta \mathrm{t}, \mathrm{c}(\mathrm{t})+\mathrm{k}_{3}\right)$

$\mathrm{c}(\mathrm{t}+\Delta \mathrm{t})=\mathrm{c}(\mathrm{t})+\frac{1}{6}\left(\mathrm{k}_{1}+2 \mathrm{k}_{2}+2 \mathrm{k}_{3}+\mathrm{k}_{4}\right)$

Where, $\Delta \mathrm{t}$ is the time step, $\mathrm{c}(\mathrm{t})$ is the concentration of the pollutant at time $\mathrm{t}, \mathrm{c}(\mathrm{t}+\Delta \mathrm{t})$ is the concentration at time $(\mathrm{t}+$ $\Delta \mathrm{t})$. The partial discretization method of the finite difference approximation of first-order spatial derivatives is:

$\frac{\partial c}{\partial x}=\frac{C_{\mathrm{i}}^{\mathrm{j}}-C_{\mathrm{i}-1}^{\mathrm{j}}}{\Delta x}$

The discrete form of derivative of the second order is:

$\frac{\partial^{2} c}{\partial x^{2}}=\frac{C_{\mathrm{i}+1}^{\mathrm{j}}-2 C_{\mathrm{i}}^{\mathrm{j}}+C_{\mathrm{i}-1}^{\mathrm{j}}}{\Delta x^{2}}$

The indices (i) and (j) represent the discretization nodes along (x) and ( $\mathrm{t}$ ) respectively. $\Delta \mathrm{x}$ is the step of space. Thus, equation (4) can be written in the following reduced form:

$\frac{\partial C_{\mathrm{i}}^{\mathrm{j}}}{\partial t}=g\left(t, x, c_{\mathrm{i}+1}^{\mathrm{j}}, c_{\mathrm{i}}^{\mathrm{j}}, c_{\mathrm{i}-1}^{\mathrm{j}}\right)$

where,

$g\left(t, x, c_{\mathrm{i}+1}^{\mathrm{j}}, c_{\mathrm{i}}^{\mathrm{j}}, c_{\mathrm{i}-1}^{\mathrm{j}}\right)=\frac{d}{\Delta x^{2}}\left(c_{\mathrm{i}+1}^{\mathrm{j}}-2 c_{\mathrm{i}}^{\mathrm{j}}+c_{\mathrm{i}-1}^{\mathrm{j}}\right)-\frac{u}{\Delta x}\left(c_{\mathrm{i}}^{\mathrm{j}}-c_{\mathrm{i}}^{\mathrm{j}}-1\right)-$ $\lambda^{\prime} c_{\mathrm{i}}^{\mathrm{j}}$

$g\left(t, x, c_{\mathrm{i}+1}^{\mathrm{j}}, c_{\mathrm{i}}^{\mathrm{j}}, c_{\mathrm{i}-1}^{\mathrm{j}}\right)=d_{1}\left(c_{\mathrm{i}+1}^{\mathrm{j}}-2 c_{\mathrm{i}}^{\mathrm{j}}+c_{\mathrm{i}-1}^{\mathrm{j}}\right)-d_{2}\left(c_{\mathrm{i}}^{\mathrm{j}}-c_{\mathrm{i}-1}^{\mathrm{j}}\right)$

$$
-\lambda^{\prime} c_{\mathrm{i}}^{\mathrm{j}}
$$

With $d_{1}=\frac{d}{\Delta x^{2}}, d_{2}=\frac{u}{\Delta x}$

We define $x=i \Delta x, t=j \Delta t$ with $\left(\mathrm{i}=0,1,2 \ldots, \mathrm{N}_{\mathrm{x}}\right)$ and $(\mathrm{j}=0$, $1,2, \ldots, \mathrm{Nt})$

The discrete version of the initial condition associated with equations (4) and (5) is expressed as follows:

$c_{\mathrm{i}}^{0}=c_{0}$ if $i=0$

$c_{\mathrm{i}}^{0}=0$ if $i \neq 0$

Equations (20) express a column without initial contamination. The discrete form of the boundary conditions is: $c_{\mathrm{i}}^{0}=c_{0}, c_{0}$ is the initial concentration of the pollutant.

\section{RESULTS AND DISCUSSION}

3.1 Spatial and temporal variation of pollutants

Figure 1 represents the spatial evolution of the concentration of the pollutant (chromium) as a function of the depth $\mathrm{x}$ for the different times $t=1$ day, $t_{1}=5$ days and $t_{2}=30$ days. $c / c_{0}$ is the ratio of the simulated value of the pollutant concentration to a depth $\mathrm{x}$. The analys is of this figure shows that at a time $t$ the concentration of the pollutant decreases exponentially inside the filter. In general, this concentration increases with the time. Considering $40 \%$ of the initial chromium concentration as a guideline, we found that the depths of the column $x$ should be $x_{1}=10 \mathrm{~cm}$ for $\mathrm{t}_{1}=1$ day, $x_{2}=20 \mathrm{~cm}$ for $\mathrm{t}_{2}=5$ days and $x_{3}=25,5 \mathrm{~cm}$ for $\mathrm{t}_{3}=30$ days.

Figure 2 shows the time evolution of the concentration of the pollutant at the filter outlet for different depth values of the filter: $\mathrm{x}_{1}=20 \mathrm{~cm}, \mathrm{x}_{2}=25 \mathrm{~cm}$ and $\mathrm{x}_{3}=30 \mathrm{~cm}$. It is found that the concentration of the pollutant increases with time and decreases as the depth of the filter increases.

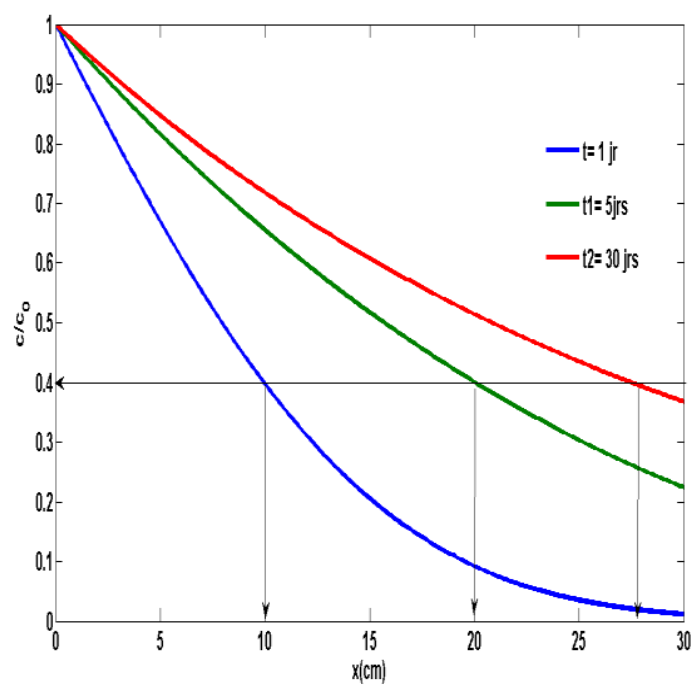

Fig. 1: Evolution of chromium concentration in space for $x$ $=30 \mathrm{~cm}$

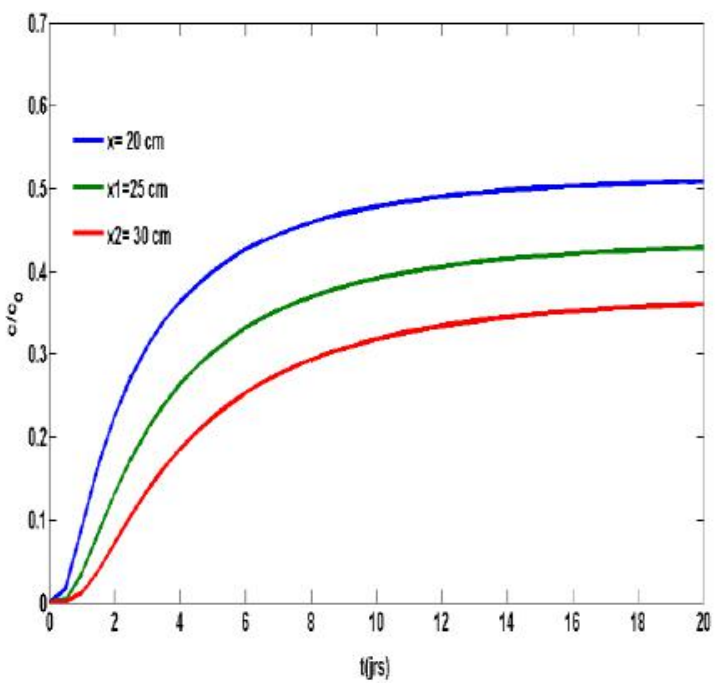

Fig. 2: Evolution of the chromium concentration over time for $t=20$ days 


\subsection{Effect of the decay rate of Chromium}

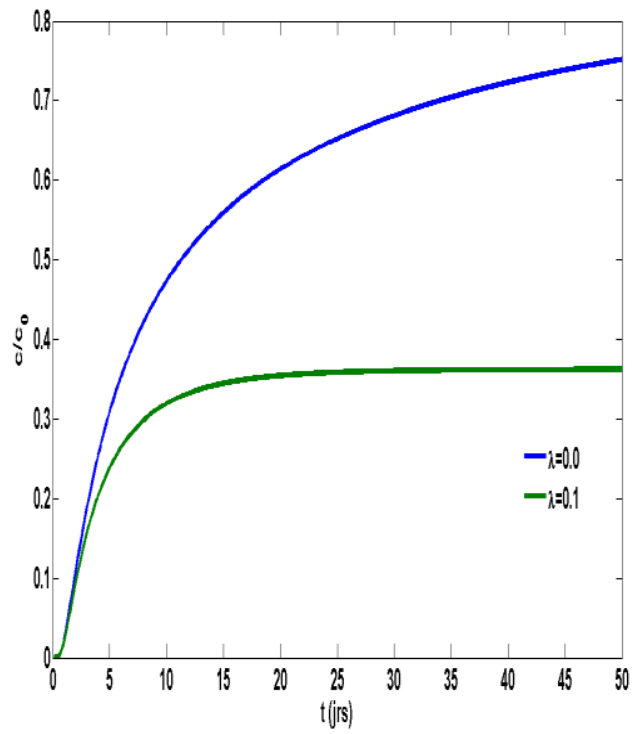

Fig. 3: the temporal evolution of the chromium concentration for the values of $\lambda=0.0$ and $\lambda=0.1$ at $t=10$ $\min$ and $x=30 \mathrm{~cm}$.

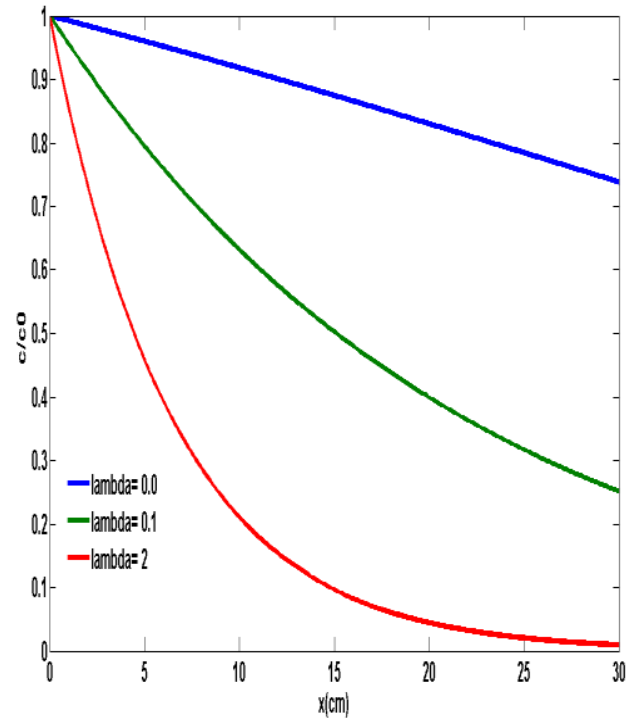

Fig. 4: the spatial evolution of the chromium concentration for the values of $\lambda=0.0$ and $\lambda=0.1$ at $t=10$ min and $x=$ $30 \mathrm{~cm}$.

Figure 3 represents the temporal evolution of the pollutant concentration in the filter with different values of the decay rate $\lambda=0.0$ and $\lambda=0.1$. We find that in the absence of this degradation coefficient i.e. when $\lambda=0$, the concentration of the pollutant evolves rapidly inside the filter, in the presence of the latter, the concentration evolves slowly.
Figure 4 represents the spatial evolution of the concentration in the filter with different values of the decay rate $\lambda=0.0,0.1$ and $\lambda=2$. The analysis of this figure reveals an exponential decay of the concentration inside the filter. The larger the lambda, the higher the concentration of the pollutant decreases rapidly inside the filter until reaching the asymptote $\mathrm{c}=0$. Therefore, the natural decay of pollutants plays an important role in wastewater treatment since it allows to avoid material waste. It is necessary for the design of industrial sorption columns

\subsection{Validation of Analytic and numerical approach}

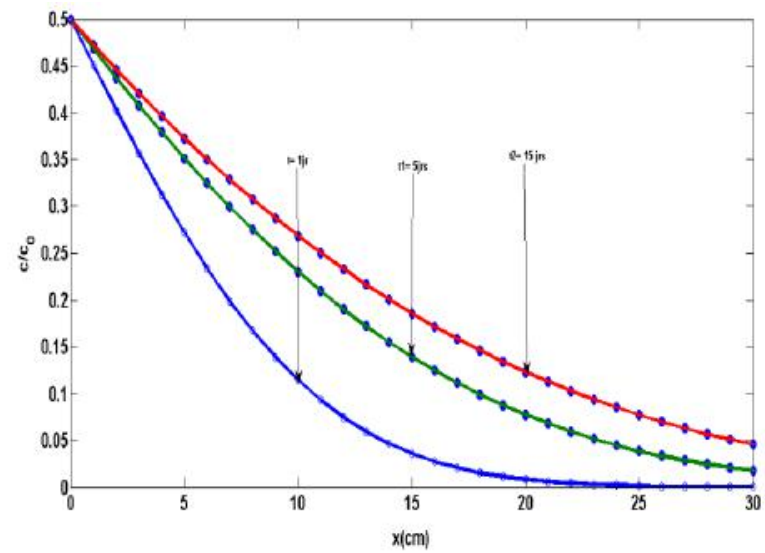

Fig.6: spatial variation of Concentration

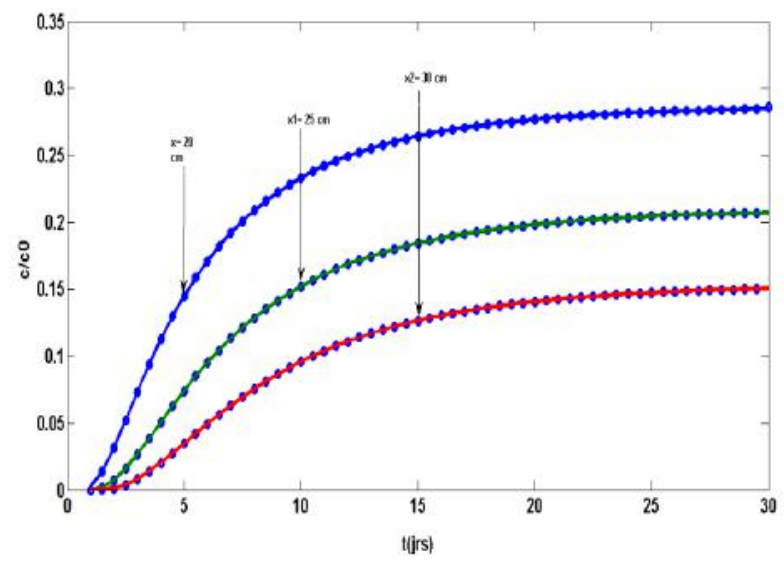

Fig.7: temporal variation of concentration

Numerical solutions of dispersion equation are generated using the RK4 algorithm. For the stability criterion, we took space step and temporal step $\Delta \mathrm{x}=1$ and $\Delta \mathrm{t}=1$ respectively. These results were obtained for different values of the filter depth $\mathrm{x}_{1}=20 \mathrm{~cm}, \mathrm{x}_{2}=25 \mathrm{~cm}$ and $\mathrm{x}_{3}=30 \mathrm{~cm}$ and for several times $\mathrm{t}_{1}=1$ day, $\mathrm{t}_{2}=5$ days and $\mathrm{t}_{3}=30$ days. After analysis, it appears on Figure 6 that the concentration of the pollutant decreases exponentially with time inside the water filter. Figure 7 shows that the concentration of the pollutant increases at the exit of the filter. The variation of the 
concentration of pollutants into filter is due to the concentration at the filter entrance and to the characteristics of the material (iron oxide-coated sand) used. Finally, the results obtained shows that these two methods (analytical and numerical) are in good agreement.

\subsection{Spatio-temporal evolution of the concentration of the pollutant}

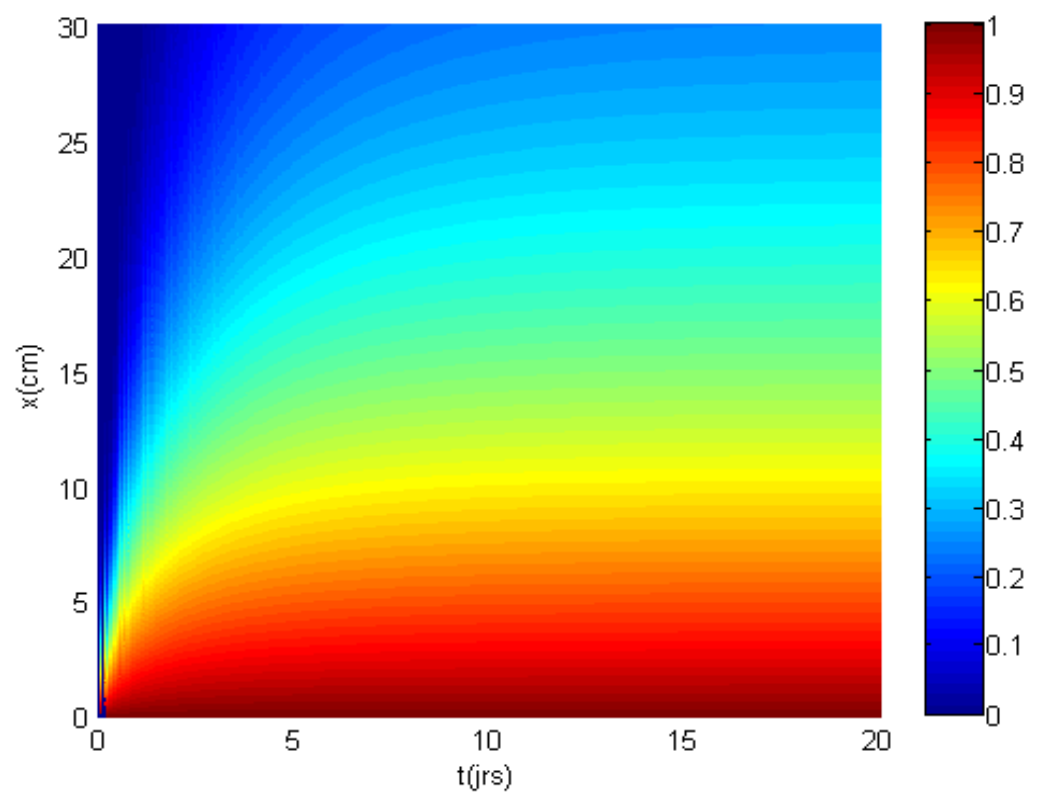

Fig.8: Spatio-temporal evolution of the pollutant concentration in the filter for $x=30 \mathrm{~cm}$ and $t=20$ days

Figure 8 shows the spatio-temporal evolution of the concentration of the pollutant in the filter. The $\mathrm{x}$-axis is as sociated to the time taken by the pollutant in the filter, and the y-axis represents the depth of the column. This figure is subdivided into three phases. The initial phase which starts from 0 to $30 \%$ of the initial concentration characterized by the blue color, the unsaturated phase from 30 to $70 \%$, and the saturated phase which start from 70 to $100 \%$ characterized by the red color. Figure 8 shows the concentration of pollutant increase with time and decrease with the column depth. These results could be used to design equipment for chromium removal in aqueous solution.

\section{CONCLUSION}

The degradation coefficient is an important factor which control the design of membrane filter to remove chromium VI in water. This coefficient must be known to optimize chromium VI adsorption in an iron oxide-coated sand based filter on a technical scale. For adsorption of chromium VI on iron oxide-coated sand, analytical and numerical data obtained from simulating a fixed bed adsorber showed that iron oxide-coated sand was very effective in removing chromium VI from an aqueous solution. It is shows that the degradation coefficient of chromium $\lambda$ has a significant effect on the spatio-temporal evolution of the concentration of the pollutant. This coefficient should be taken into account in models in order to avoid to overestimate the concentration of the pollutant in reactive media. A clear improvement is observed for a material having a high adsorption capacity and a high first order degradation coefficient. Affordable water treatment filters could be cons tructed using iron oxide-coated sand which is an effective, inexpensive material for the removal of water pollutants or for the treatment of effluents from industries.

\section{ACKNOWLEDGMENTS}

Thanks are due to our colleagues from the Environmental physics laboratory, Faculty of Science, University of Yaoundé I, Cameroon for their direct and indirect help for the completion of research and for technical support.

\section{CONFLICTS OF INTEREST}

The authors declare no conflict of interest.

\section{REFERENCES}

[1] Ali, I., Gupta, V.K. (2007). Advances in Water Treatment by Adsorption Technology, Nature Protocols. 1: 2661-2667.

[2] WHO/UNICEF (2008). Joint monitors program for water supply and sanitation; World Health Organization Press, Geneva, Switzerland. 
[3] Ahamed, S., Munir, A. K. M., Hussan, A. (2009). Groundwater arsenic removal technologies based on sorbents: Field applications and sustainability. In Handbook of water quality and water purity, Elsevier Inc., chapter 16, 379.

[4] Tchobanoglous, G., Burton, F.L. (1991). Wastewater Engineering, Management. 7: 1-4.

[5] Desjardins, R. (1997). Le traitement des eaux. Presses Internationales Polytechnique, Montréal, Canada.

[6] Joshi, A., Chauduri, M. (1996). Removal of Arsenic from ground water by iron oxide-coated sand, Journal of environmental engineering . 1: 769-771.

[7] Bailey, R.P., Bennett, T., Benjamin, M. M. (1992). Sorption onto and Recovery of Cr (VI) Using IronOxide-Coated Sand, Water Sci Technol. 26: 1239-1244.

[8] Williams, L. E., Barnett, M. O., Kramer, T. A., Melville, J. G. (2003). Adsorption and Transport of Arsenic (V) in Experimental Subsurface Systems, Journal of Environment Quality. 32:841-850.

[9] Runkel, R. L. (1996). Solution of the advectiondispersion equation: continuous load of finite duration, Journal of Environmental Engineering.122: 830-832 